\title{
Zone Plate Performance as a Function of Tilt Analyzed via Multislice Simulations
}

Syed Sajid $\mathrm{Ali}^{1}{ }^{1}$, Kenan $\mathrm{Li}^{2}$, Michael Wojcik ${ }^{3}$ and Chris Jacobsen ${ }^{3,4,5 *}$

1. Applied Physics, Northwestern University, Evanston, IL USA.

2. SLAC National Accelerator Laboratory, Menlo Park, CA USA.

3. Advanced Photon Source, Argonne National Laboratory, Lemont, IL USA.

4. Department of Physics \& Astronomy, Northwestern University, Evanston, IL USA.

5. Chemistry of Life Processes Institute, Northwestern University, Evanston, IL USA.

* Corresponding author, c-jacobsen@u.northwestern.edu

Thanks to new nanofabrication processes for hard x-ray Fresnel zone plates [1,2], the finest zone width (required for high spatial resolution) has been decreasing while the thickness (required for diffraction efficiency) has been increasing. This leads to higher aspect ratios, with values as high as 500:1 having been demonstrated [2]. At these aspect ratios, one must consider carefully the effects of small tilt misalignments of a zone plate relative to the $\mathrm{x}$-ray beam. Several estimates of this tilt tolerance have been provided based on the simple analysis of optical path lengths for thin zone plates [3], with a more recent estimate [4] being based on considering depth of focus limits. However, zone plates with very high aspect ratios, and finest zone widths that begin to approach a small multiple of the $\mathrm{x}$-ray wavelength, need a more sophisticated approach: they begin to act as volume gratings [5], and these waveguide-like effects must be taken into account.

While coupled wave theory has been used to understand the performance of thicker zone plates [5], it is usually used under the approximation of calculating the diffraction efficiency of zones with locally constant grating period. However, in a tilted zone plate one must consider the net optical effect of all zone periods on the focus. We have therefore used a multislice approach to consider the optical performance of high aspect ratio zone plates as a function of tilt. In the multislice approach [6], an object is conceptually "sliced up" in to thin slabs; one applies the refractive index effects of the slab on the wavefield, which is then propagated to the next slab position. This approach has been shown to reproduce coupled wave theory results for x-ray nanofocusing [7], provided one uses a weighted filling of voxels at boundaries between zone materials. For our calculations, we discretize a non-tilted zone plate, and consider incident beams at non-orthogonal angles.

We have studied the dependence on tilt while varying the thickness of the zone plate and the number of zones. The number of steps used for propagation was decided according the metric given in [7]. We used a grid of $45 \mathrm{k}$ by $45 \mathrm{k}$ pixels and input pixel size of $3.3 \mathrm{~nm}$ and output pixel size of $5.3 \mathrm{~nm}$. All calculations performed thus far are at $10 \mathrm{keV}$ with $20 \mathrm{~nm}$ outermost zone width and a focal length of $6.45 \mathrm{~mm}$, though a wider range of parameters is planned for future work.

We observe that the performance of a zone plate versus tilt angle varies both as a function of number of zones and the thickness of a zone plate. Two examples are shown below. We can clearly see that thicker zone plates have a much tighter tilt tolerance and need to be mounted accordingly at the beamline for optimum performance. 
References:

[1] K Jefimovs et al., Phys. Rev. Lett. 99 (2007), 264801; C. Chang and A. Sakdinawat, Nat. Commun. 5 (2014), 4243

[2] K. Li et al., J. Vac. Sci. Technol. B 35 (2017) 06G901.

[3] O. Myers, Am. J. Phys. 19 (1951), 359; M Young, J. Opt. Soc. Am. 62 (1972), 972

[4] C. Jacobsen et al., Opt. Comm. 86 (1991), p 351.

[5] J. Maser and G. Schmahl, Opt. Comm. 89 (1992), 355; G. Schneider, Applied Physics Letters 71 (1997), 2242; G. Schneider et al., in "Modern Developments in X-ray and Neutron Optics," ed. A. Erko et al., (Springer, Berlin, 2008), 137.

[6] J M Cowley et al, Acta Cryst. 10 (1957), 609.

[7] K. Li and C. Jacobsen, Opt. Express 25 (2017), 185.

[8] This work is supported by NIH under grant U01 MH109100, and US DoE Office of Science under contract DE-AC02-06CH11357 to ANL. Code available at: https:/github.com/s-sajidali/zone_plate_testing/releases/tag/0.1.0
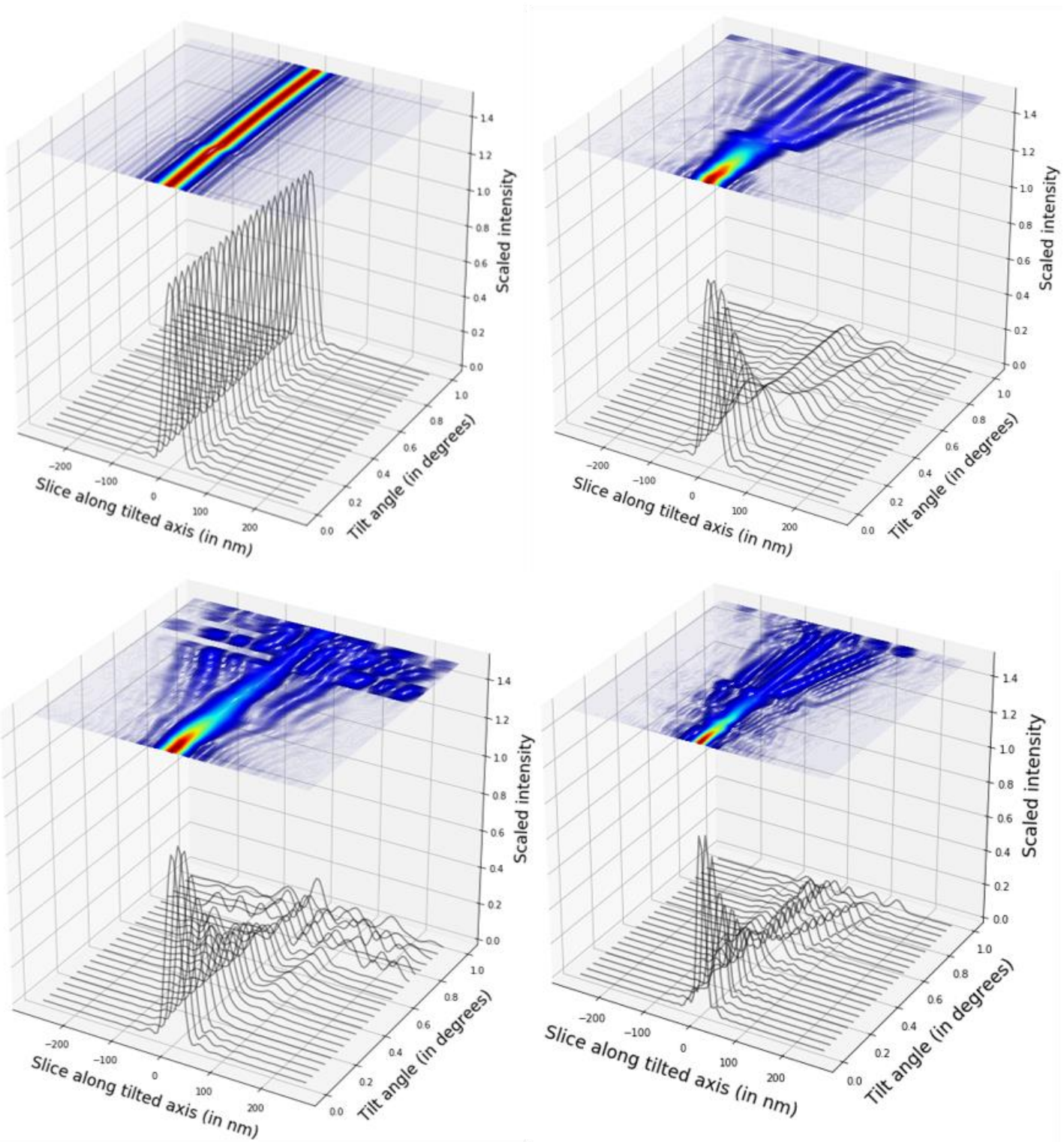

Figure. 1. Zone plate focal profiles along the axis of tilt for four cases. A filled contour plot is added to each for ease of interpretation. Top: 200 zones, 0.5 and 10 microns. Bottom: 8 microns, 200 and 